\title{
A Review on Antiplatelet Drugs and Anticoagulants
}

Jhansi Konduru and Vanita $P$

Department of Biochemistry, Dr. L.B. College, Visakhapatnam, India

Corresponding author: Jhansi Rani Konduru, Department of Biochemistry, Dr. L.B. College, Visakhapatnam, India, Tel: +91-9885352429; E-mail: kondurujhansi68@gmail.com

Copyright: (C) 2014 Jhansi K, et al. This is an open-access article distributed under the terms of the Creative Commons Attribution License, which permits unrestricted use, distribution, and reproduction in any medium, provided the original author and source are credited

\section{Abstract}

Antiplatelet Drugs and Anticoagulants drugs help prevent dangerous blood clots from forming. These are used to control the risk heart attack or stroke, in these cases they want to use by doctors prescription. These are very harmful to the body, so that people will change the lifestyle when they have to maintain good health to control it.

Keywords Antiplatelet Drugs; Anticoagulants; Blood clot; Stroke; Heart attack

\section{Introduction}

Anticoagulants and antiplatelet agents are drugs used to reduce the risk of blood clots or prevent existing clots from getting larger in the body. These are also known as blood thinners. These medicines help to prevent the clots the in blood vessels or heart [1]. When get the cut or scarp blood clots are commonly good. But, when blood clots form in the blood vessels, arteries or heart, these are very dangerous because they can block the blood circulation. Ultimately they stop the flow of blood. These blocks can cause the heart attack and also block the blood in brain, it causing stroke. Certain types of heart valves are prone to forming blood clots. Such clots can shoot out to the lungs or the brain. Which is called preventive or prophylactic treatment. Anticoagulant and antiplatelet drugs work by preventing the platelets from following to one another and clotting proteins from binding together [2]. Oral antiplatelet agents have been reported to reduce vascular deaths by $15 \%$ and serious cardiovascular events by $20 \%$ in high risk persons [3]. The effect of antiplatelet therapy in diabetic neuropathy should be examined in long-term clinical trials that may potentially unfold its benefits over time [4].

\section{Types of Anticoagulant and Antiplatelet Drugs}

1. There are two types of anticoagulant drugs commonly used Heparin

Coumadin

\section{Commonly used Antiplatelet Drugs drugs are}

Aspirin: Acetylsalicylic acid or Aspirin (ASA) is a over pain reliever that also has anti platelet properties. Aspirin is a "classic" antiplatelet agent frequently used in primary and secondary prevention of atherothrombotic events not only in patients with ACS. Large numbers of patients continue to experience these events despite aspirin therapy [5]

Clopidogrel: Clopidogrel (clopidogrel bisulfate, Plavix) is an antiplatelet drug that is effective is reduce the strokes and heart attacks and is often prescribed to patients who receive a coronary stent. Clopidogrel is a pro-drug that requires hepatic biotransformation [6].
Rotational atherectomy allows plaque remodeling in severely calcified lesions and, prior to the adoption of clopidogrel as standard therapy [7].

Prasugrel: Prasugrel is also related to Clopidogrel, it is a novel prodrug from the same family as clopidogrel, with more efficient platelet inhibition.

Dipyridmalole: Dipyridmalole is commonly used as anti-platelet or anticoagulant drugs, Acetylsalicylic acid or Aspirin. It can also be given by injection during tests on the heart to the patients.

Glycoprotein IIb/IIIa antagonists: Abciximab was the original GP IIb/IIIa antagonist, thase are a monoclonal antibody with a much continued duration compared to newer agents, eptifibatide is a nonpeptide antagonist. These drugs prevent the final common pathway of platelet formation where fibrinogen binds to GP IIb/IIIa receptor [8]

Thienopyridines: The antiplatelet activities of the indirectly acting thienopyridines are delayed as a consequence of the need for metabolism of the prodrug. Furthermore, there are considerable interindividual discrepancies in the degree of metabolism of the prodrug leading to unpredictability in platelet inhibition [9].

\section{How does it work?}

Antiplatelet drugs are intended to prevent and/or reverse platelet aggregation in arterial thrombosis, most prominently in myocardial infarction and ischaemic stroke.

Anti-platelets, such as Acetylsalicylic acid or Aspirin (ASA), act on platelets in the blood, assemble it less sticky so that the platelets are less possible to produce clots that block arteries and may cause heart attacks or strokes [10]. Dual antiplatelet therapy (DAPT) consisting of aspirin and a thienopyridine was used to mitigate any negative consequences including stent thrombosis and recurrent cardiovascular events [11].

\section{Difference}

Anticoagulates such as heparin or warfarin, those are most effective for involving venous clots. When an excessive amount of fibrin venous clots are formed, Fibrin protein that causes blood clotting, it develops in blood. An anticoagulant use to dissolves venous clots by disrupting the development of fibrin protein, it is the best drug choice for conditions such as deep-vein thrombosis or pulmonary embolism. In 
contrast, Antiplatelets are not generally suitable for using to treat the venous clots and are these most effective for preventing artery clots. When damage occured Platelets are join together in the blood, such as cuts, bruises or internal injuries occurs. Sometimes Platelets also combine too rapidly when the arteries are inflamed, causing a block in an artery wall. Antiplatelet drugs such as aspirin, prevent the platelets from forming and are usually these are prescribed when clots are artery-based.

Antiplatelets target the production of thromboxane, these are secreted by platelets that causes blood clotting, Anticoagulants attack only clotting factors. These are any other agents that contribute to blood clots. Anticoagulants are generally stronger and more aggressive than Antiplatelets [12].

Advantages

- The advantages and limitations of various antiplatelet agents that have been approved by the US Food and Drug Administration (FDA) or under development [13].

- The advantages are that they no longer require blood tests to monitor their effectiveness

- The greatest advantage of these drugs is that they prevent blood clots and also the serious complications. Aspirin specially is a lifesaver. Reduce the risk of a transient ischemic attack (TIA), stroke, and heart attack.

- a short course of anticoagulation is good enough. Typically, the longer a person is on blood thinners, the less their chance of having another clot, but this must be weighed against the increase chance for bleeding. After a second clot, though, sometimes lifelong is better.

- Anticoagulants carry a risk of bleeding. Usually there is a very good reason for taking anticoagulants in pregnancy.

- Anticoagulant medication is not used to treat rheumatoid arthritis.

\section{Disadvantages}

- Typically the drugs will cause harm issues, headaches, dizziness, pain, discomfort. gastritis, ulceration, and bleeding [14,15]

- ASA can irritate your stomach and in some cases can cause bleeding. ASA should never be given to children because it has been linked to Reye's syndrome, a rare brain and liver disorder.

- Coumadin can cause the risk for bleeding, pregnant, or breastfeeding. Because anti-platelets interfere with the clotting action of the blood, they can increase the risk of uncontrolled bleeding. Anti-platelets should not be taken by individuals who have an ulcer or history of bleeding problems. Abnormal bruising may be a symptom of a bleeding problem. Anti-platelet medications can irritate the stomach, promote the development of ulcers, and increase water and salt retention.

- It is high cost \& safety concerns

- Enteric coated or buffered tablets have not been shown to reduce the likelihood of major gastrointestinal adverse effects.
- Increased risk of bleeding in surgical procedures due to impaired primary hemostasis.

- Decrease the Platelet's life span

\section{Conclusion}

The present observations on Anticoagulants and antiplatelet agents are partially useful and cause so many side effects in the body. So the people will change the everyday lifestyle. Eating a healthy diet that's lower in fat, particularly saturated and trans fats, being smoke free, limiting alcohol use, being physically active and reducing stress also are vital in lowering the chance of heart condition. Visit your healthcare practician concerning however you'll be able to come through these life style changes. Hence these should be used under proper guidance of the physician. This will reduce the side effects of these birth control drugs. The routine transfusion of platelets in TBI patients on preinjury antiplatelet therapy [16]

\section{References}

1. https://www.heart.org/idc/groups/heart-public/@wcm/@hcm/documents/downloadable/ ucm_300338.pdf

2. http://www.healthline.com/health/anticoagulant-and-antiplatelet-drugs

3. Fourtounas C, Panagiotou A (2013) Oral Antiplatelet Agents in Dialysis Patients: Friends or Foes? General Med 1:109. doi: 10.4172/2327-5146.1000109

4. Deguchi T, Rosales RL, Hashiguchi T, Arimura K (2012) Antiplatelet Therapy, Diabetic Neuropathy and Peripheral Vascular Disease: A Unitary Approach. J Diabetes Metab S5:005. doi: 10.4172/2155-6156.S5-005

5. Samos M, Fedor M (2014) Monitoring of Antiplatelet Therapy in Clinical Practice: Is it Necessary or Not? J Hematol Thromb Dis 2: 129. doi: 10.4172/2329-8790.1000129

6. Lee VWY (2013) Pharmacogenomic Testing of Anti-Platelets Drugs and its Cost-Effectiveness. J Clin Toxicol 3:e120. doi: 10.4172/2161-0495.1000e120

7. García-Lara J, Pinar-Bermúdez E, Lacunza-Ruiz J, Valdesuso-Aguilar R, Hurtado JA, et al. (2013) Lack of Peri-Procedural Myocardial Necrosis Reduction from Abciximab in Patients on Dual Antiplatelet Therapy Revascularized with Rotational Atherectomy. J Clin Exp Cardiolog 4:270. doi: 10.4172/2155-9880.1000270.

8. Fourtounas C, Panagiotou A (2013) Oral Antiplatelet Agents in Dialysis Patients: Friends or Foes? General Med 1:109. doi: 10.4172/2327-5146.1000109

9. Khalil MZ (2013) Role of Antiplatelet Therapies in Preventing Atherothrombosis. J Hematol Thromb Dis 1:108. doi: 10.4172/2329-8790.1000108

10. http://www.patient.co.uk/doctor/Anti-platelet-Drugs.htm

11. Gillette MA (2013) Antiplatelet Development: The Search for America's Next Top Agent. J Develop Drugs 2: e119. doi: 10.4172/2329-6631.1000e119

12. http://www.heartandstroke.com/site/c.ikIQLcMWJtE/b.3484139/k.C87F/ Heart_disease_Antiplatelets.htm

13. Jing F, Zhang W (2013) Thrombosis Therapy: Focus on Antiplatelet Agents. Int J Genomic Med 1:103. doi: $10.4172 / \mathrm{ijgm} .1000103$

14. http://www.ehow.com/list_7354770_antiplatelet-anticoagulant-differences.html

15. http://www.healio.com/orthopedics/journals/ortho/\%7Bc3ff2694-5e5a-4517-ade4bd2131df4461\%7D/optimizing-antiplatelet-and-anticoagulant-agents-in-the-perioperativeorthopedic-surgery-patient

16. Sadaka F (2014) Traumatic Brain Injury and Preinjury Antiplatelet Use: Is Platelet Transfusion Helpful? J Blood Disorders Transf 5:e113. doi: 10.4172/2155-9864.1000e113 